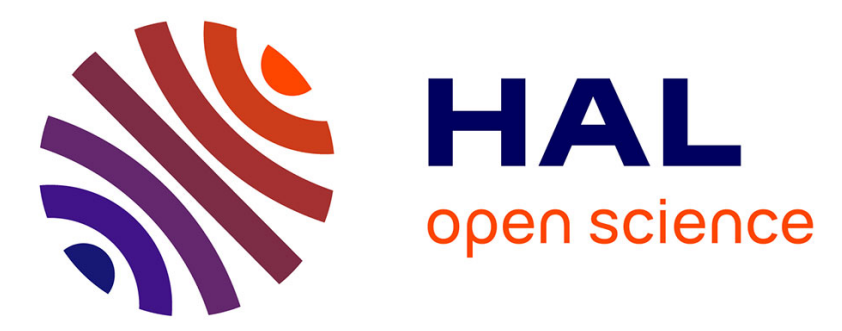

\title{
ESTIMATION DU CHAMP ACOUSTIQUE LOINTAIN D'UNE PLAQUE PLANE BAFFLÉE À PARTIR DE LA MESURE DE SON CHAMP D'ACCÉLÉRATION
}

\author{
D. Blacodon, D. Brenot
}

\section{To cite this version:}

D. Blacodon, D. Brenot. ESTIMATION DU CHAMP ACOUSTIQUE LOINTAIN D'UNE PLAQUE PLANE BAFFLÉE À PARTIR DE LA MESURE DE SON CHAMP D'ACCÉLÉRATION. Journal de Physique IV Proceedings, 1992, 02 (C1), pp.C1-507-C1-510. 10.1051/jp4:19921109 . jpa-00251063

\section{HAL Id: jpa-00251063 https://hal.science/jpa-00251063}

Submitted on 1 Jan 1992

HAL is a multi-disciplinary open access archive for the deposit and dissemination of scientific research documents, whether they are published or not. The documents may come from teaching and research institutions in France or abroad, or from public or private research centers.
L'archive ouverte pluridisciplinaire HAL, est destinée au dépôt et à la diffusion de documents scientifiques de niveau recherche, publiés ou non, émanant des établissements d'enseignement et de recherche français ou étrangers, des laboratoires publics ou privés. 
ESTIMATION DU CHAMP ACOUSTIQUE LOINTAIN D'UNE PLAQUE PLANE BAFFLEE À PARTIR DE LA MESURE DE SON CHAMP D'ACCÉLÉRATION

\author{
D. BLACODON et D. BRENOT
}

Office National d'Etudes et de Recherches Aérospatiales, BP. 72, F-92322 Châtillon cedex, France

\title{
RESUME
}

Les méthodes de prévision du bruit rayonné par les structures vibrantes ont un grand intérêt dans de nombreuses applications pratiques. Quand une mesure de l'accélération pariétale est effectuée, elle peut être utilisée avec une méthode qui s'appuie sur le calcul de l'intégrale de Rayleigh, pour estimer le niveau sonore émis en champ lointain. On donne, dans cet article, une formulation de la méthode de prévision, puis on montre qu'elle fournit des résultats corrects jusqu'à une fréquence qui dépend de l'échantillonnage spatial du champ d'accélération. Les résultats d'une expérience avec une plaque d'acier sont fournis et discutés.

\section{ABSTRACT}

Prediction methods of noise radiated by vibrating structures are of a great interest in numerous actual situations. Far-field sound prediction may be performed from the measurements of structure acceleration, associated with a method based on the Rayleigh integral. A formulation of this method is given in the paper. In practice, the acceleration distribution is sampled by an array of accelerometers, it is shown that the method works up to a frequency dependent on the spatial sampling. Results and an experiment with a steel baffled plate are given and discussed.

\section{LISTE DE NOTATIONS}

C : célérité du son,

f : fréquence,

J,D : nombre de lignes et colonnes de la matrice d'accéléromètres,

$k=2 \pi f / c \quad:$ nombre d'ondes acoustiques,

$k_{x}, k_{y} \quad:$ nombres d'ondes longitudinaux et transversaux de structure,

$L_{x}, L_{y} \quad$ : dimensions de la plaque,

$\vec{r} \quad$ : point d'observation de coordonnées $r, \theta, \varphi$,

$\rho_{0} \quad$ : masse volumique de l'air,

$\gamma(x, y, f)$ : transformée de Fourier à la fréquence f de l'accélération pariétale $\gamma(x, y, f)$.

\section{INTRODUCTION}

Les méthodes d'estimation du bruit rayonné par les structures ont un grand intérêt dans de nombreux cas pratiques en aéroacoustique ainsi qu'en hydroacoustique. Elles sont communément utilisées pour évaluer en champ lointain les caractéristiques énergétiques des modes de structure engendrés par une excitation de type mécanique aéro- ou hydrodynamique.

Pour effectuer cette opération, certaines méthodes d'estimation, décrites dans [1, 2], s'appuient sur une mesure de pression pratiquée en général dans le champ proche des sources acoustiques. L'approche retenue dans cet article 
est différente, puisque la méthode d'estimation étudiée est bâtie sur une mesure du champ d'accélération pariétal par une matrice d'accéléromètres.

Après un rappel de la formulation théorique de la méthode d'estimation, on montre que la fréquence maximale de prévision est fonction de l'échantillonnage spatial du champ d'accélération. La méthode a été essayée sur des données issues d'une expérience qui a eu lieu dans la chambre anéchoïque de l'ONERA Palaiseau avec une plaque d'acier bafflée. On décrit les configurations étudiées, l'instrumentation utilisée pour la mesure de l'accélération pariétale et pour le champ lointain. Finalement, on effectue l'analyse de certains résultats de mesure et de calcul.

\section{PRESSION ACOUSTIQUE RAYONNEE EN CHAMP LOINTAIN PAR UNE PLAQUE PLANE BAFFLEE}

La géométrie du problème est décrite sur la figure 1 . On considère une plaque plane, définie par $-L_{x} / 2 \leqslant x \leqslant L_{x} / 2$, $-L / 2 \leqslant y \leqslant L / 2$, située dans un baffle parfaitement rigide dans le plan $z=0$. On s'intéresse au calcul de la pression sonore qu'elle rayonne en champ lointain pour chaque fréquence, à partir d'une mesure de l'accélération pariétale. La solution à ce problème est fournie par l'intégrale de Rayleigh [3]. En champ lointain, elle se met sous la forme suivante :

$$
\nu(r, \theta, \varphi f)=\frac{\rho_{0} \cdot e^{i b r}}{2 \pi r} \int_{-\frac{L_{x}}{2}}^{\frac{L_{x}}{2}} \int_{-\frac{L_{y}}{2}}^{\frac{L_{y}}{2}} e^{\left.-\| L_{[x} \cdot k_{x}+y \cdot k_{y}\right]} \cdot \gamma(x, y, f) d x \cdot d y,
$$

avec $k_{x}=k \cdot \sin \theta \cdot \cos \varphi$ et $k_{y}=k \cdot \sin \theta \cdot \sin \varphi$.

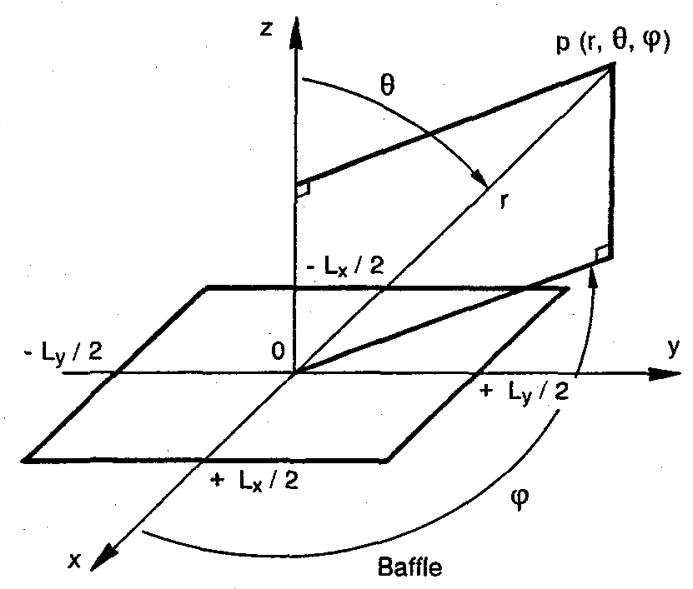

Fig. 1 - Géométrie du problème du calcul de la pression rayonnée en champ lointain par une plaque bafflée.

\section{FREQUENCE MAXIMALE DE PREVISION}

La méthode d'estimation a pour donnée d'entrée le champ d'accélération pariétal $\gamma(x, y, f)$. Dans la présente application, celui-ci est échantillonné spatialement aux positions $-L_{x} / 2+j \Delta_{x}\left(j=0,1,2, \ldots, J-1 ; \Delta_{x}=L_{x}(J-1)\right)$, $-L_{\gamma} / 2+d \Delta_{y}\left(d=0,1,2, \ldots, D-1 ; \Delta_{y}=L_{y}(D-1)\right)$. En conséquence, les nombres d'ondes $k_{x}, k_{y}$ que contient $\gamma(x, y, f)$ doivent être suffisamment bas pour que le théorème de Shannon soit respecté. Lorsque cette condition est remplie, il est possible de déterminer la fréquence maximale de prévision de la méthode. En effet, pour éviter les problèmes de repliement spatial lors du calcul de l'expression (1), les nombres d'ondes $k_{x}, k_{y}$ définis par la relation (2) doivent vérifier :

$$
\left\{\begin{array}{lll}
|k \cdot \sin \theta \cdot \cos \varphi| \leq 2 \pi \bar{k}_{x \max } & \text { avec } & \bar{k}_{x \max }=\frac{1}{2 \Delta x} \\
|k \cdot \sin \theta \cdot \sin \varphi| \leq 2 \pi \bar{k}_{y \max } & \text { avec } \bar{k}_{\mathrm{ymax}}=\frac{1}{2 \Delta y}
\end{array}\right.
$$


Sachant que $k=2 \pi i c$, il vient :

$$
\left\{\begin{array}{l}
f_{x} \leq \frac{c \cdot \bar{k}_{x \max }}{|\sin \theta \cdot \cos \varphi|} \\
f_{y} \leq \frac{c \cdot \bar{k}_{y \max }}{|\sin \theta \cdot \sin \varphi|}
\end{array}\right.
$$

Finalement, la valeur recherchée est donnée par :

$$
f_{\max } \leq \min \left(f_{x}, f_{y}\right)
$$

Cette expression montre que la pression sonore (1) est estimée correctement jusqu'à une fréquence $f_{\max }$ qui dépend à la fois de l'échantillonnage spatial de l'accélération pariétale et des angles sphériques $\theta$ et $\varphi$ de la direction d'observation.

\section{DESCRIPTION DE L'EXPERIENCE}

La méthode d'estimation est essayée sur les données provenant d'une expérience avec une plaque d'acier de dimensions $L_{x}=28 \mathrm{~cm}, L_{y}=49 \mathrm{~cm}$ et d'une épaisseur $h=1,2 \mathrm{~cm}$. Elle est insérée dans un baffle constitué de plaques de polystyrène expansé, sur lesquelles sont posés des panneaux de bois recouverts d'une peau acoustiquement réfléchissante.

Pendantl'expérience, la plaque est soumise à une excitation ponctuelle créée par un pot vibrant. Dans une première configuration, la position du pot est au centre de la plaque (en $x=0=y=0$ ) afin qu'un faible nombre de modes propres soit excité : les modes d'indices $(\mathrm{m}, \mathrm{n})$ impairs. Dans une seconde configuration, il est décentré en $\mathrm{x}=-6 \mathrm{~cm}$, $y=-14 \mathrm{~cm}$ afin qu'un grand nombre de modes soit excité : les modes d'indices $(m, n)$ pairs et impairs.

Pour effectuer la mesure de l'accélération pariétale, on utilise une matrice de quatre rangées de sept accéléromètres Bruel \& Kjaer 4374. Le champ lointain est exploré par huit microphones Aksud 4133 situés sur un support semi-circulaire d'un rayon de $2 \mathrm{~m}$. Pour chacune des deux positions du pot vibrant, le support est déplacé autour de son axe, permettant ainsi la mesure du rayonnement de la plaque sur une demi-sphère.

\section{SPECTRE DU SIGNAL D'UN ACCELEROMETRE}

La figure 2a présente le spectre du signal d'un accéléromètre lorsque le pot vibrant est au centre de la plaque. II met en évidence de nombreuses raies qui correspondent aux fréquences propres de la plaque. Les premières raies, aux fréquences de $450,1250,2850$ et $3350 \mathrm{~Hz}$, correspondent à l'excitation des modes propres $(m=1, n=1)$, $(m=1, n=3),(m=1, n=5),(m=3, n=1)$. Comme prévu, les modes propres d'indices pairs sont faiblement excités.

dB (réf. $10^{-4}$ m.s-2)

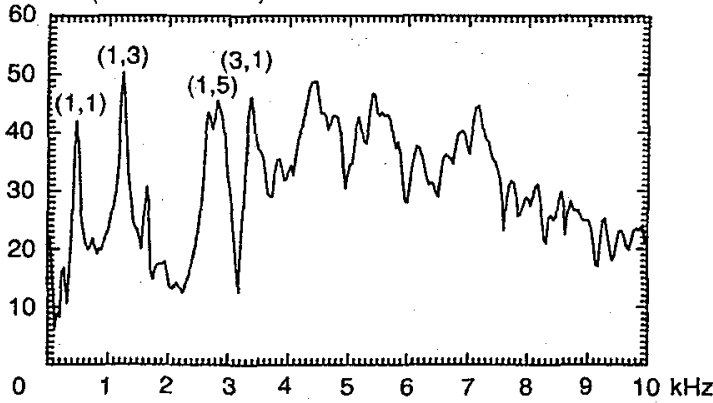

(a)
dB (réf. 10-4 m.s.2)

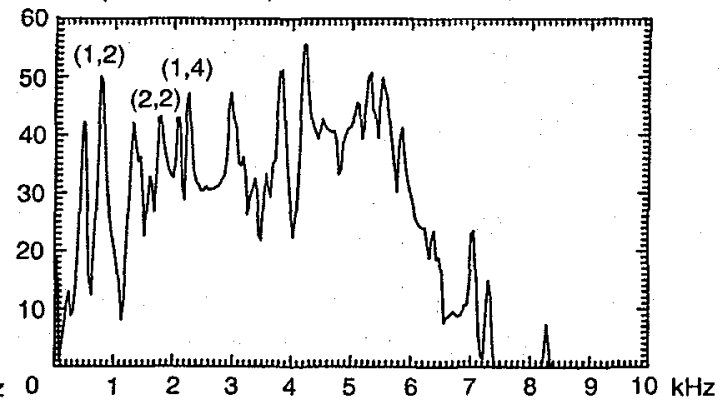

(b)

Fig. 2 - Spectre du signal d'un accéléromètre :

a) pot vibrant au centre de la plaque,

b) pot vibrant décentré. 
La figure $2 b$ présente le spectre du même accéléromètre avec le pot vibrant décentré. La décroissance du niveau du spectre en haute fréquence est due au filtre anti-repliement, dont la fréquence était fixée ici à $5 \mathrm{kHz}$. Comme on pouvait s'y attendre, les raies sont plus nombreuses car les modes propres d'indices pairs et impairs sont excités. On remarque notamment que les modes propres $(m=1, n=2),(m=2, n=2),(m=1, n=4)$ sont excités aux fréquences de 800,1800 et $2100 \mathrm{~Hz}$.

\section{COMPARAISON DES NIVEAUX SONORES CALCULES ET MESURES EN CHAMP LOINTAIN}

Afin d'analyser aisément les deux types de résultats, on représente sur une même figure le spectre mesuré (en trait pointillé) et le spectre estimé (en trait plein) dans la bande 0 à $10 \mathrm{kHz}$. On se limite ici à la comparaison des spectres obtenus aux points situés en $\mathrm{P}_{1}\left(\theta=75^{\circ}, \varphi=30^{\circ}, \mathrm{r}=2 \mathrm{~m}\right)$ lorsque le pot vibrant est centré et en $\mathrm{P}_{2}\left(\theta=-30^{\circ}, \varphi=90^{\circ}\right.$, $r=2 \mathrm{~m}$ ) quand il est décentré.

On observe un très bon accord entre les résultats jusqu'à une fréquence $f=2903 \mathrm{~Hz}$ pour le point $P_{1}$ (fig. $3 a$ ) et jusqu'à $f=4857 \mathrm{~Hz}$ pour le point $P_{2}$ (fig. $3 \mathrm{~b}$ ). Au-delà de ces fréquences, le niveau du spectre estimé est très supérieur à celui du spectre mesuré pour la raison évoquée au paragraphe 3 . Les valeurs de $f_{\max }$, tirées de l'expression (5), coïncident avec les valeurs expérimentales. Ce résultat montre que le domaine de validité de la méthode d'estimation dépend effectivement de l'échantillonnage spatial et de la direction d'observation.

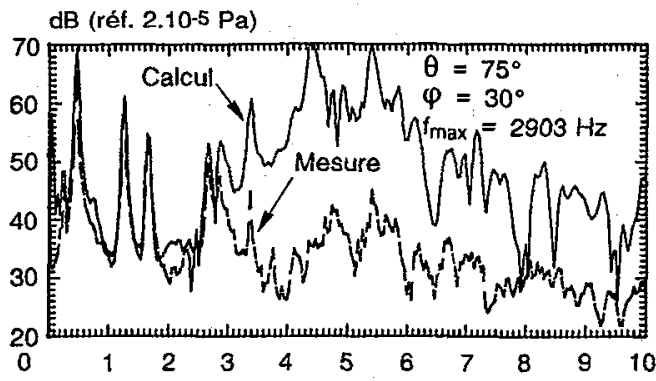

(a)

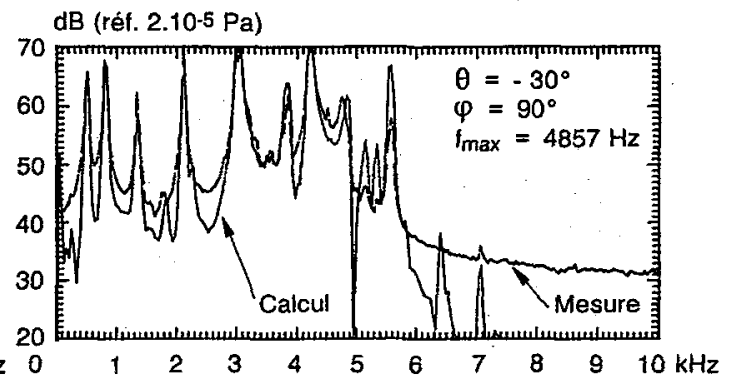

(b)

Fig. 3 - Spectres calculés et mesurés :

a) au point $P_{1}\left(\theta=75^{\circ}, \varphi=30^{\circ}, r=2 \mathrm{~m}\right)$ lorsque le pot vibrant est centré,

b) au point $P_{2}\left(\theta=-30^{\circ}, \varphi=90^{\circ}, r=2 \mathrm{~m}\right)$ lorsque le pot vibrant est décentré.

\section{CONCLUSION}

Les résultats présentés ont permis, d'une part, de valider une méthode d'estimation fondée sur une mesure de l'accélération pariétale et, d'autre part, de vérifier que la fréquence maximale de prévision est fonction de l'échantillonnage spatial.

\section{REMERCIEMENTS}

Ce travail a été réalisé sous contrat DRET et a bénéficié de nombreux échanges avec $G$. Elias et A. Julienne. La mise en place de l'expérience a été assurée par J. Chombart et F. Remblière.

\section{REFERENCES}

[1] - S.M. Candel, Radial extrapolation of wave fields by spectral methods, J. Acoust. Soc. Am., 76, 1823-1828, 1984.

[2] - G. Elias, Far-fields extrapolation of near-field measurement using inverse method, AlAA Paper No. 89-1115, 1989.

[3] - M.C. Junger, Sound, structures and their interaction, The M.IT. Press, Cambridge, London, 1972. 